\title{
Directionality in scattering by nanoparticles: Kerker's null-scattering conditions revisited
}

\author{
B. García-Cámara, * R. Alcaraz de la Osa, J. M. Saiz, F. González, and F. Moreno \\ Universidad de Cantabria, Grupo de Optica, Departamento Fisica Aplicada, \\ Facultad de Ciencias, Avenida Los Castros S/N, Santander, Spain \\ *Corresponding author: garciacb@unican.es
}

Received September 30, 2010; revised January 27, 2011; accepted February 3, 2011; posted February 4, 2011 (Doc. ID 135966); published February 28, 2011

\begin{abstract}
Since the first studies made by Kerker in the 1970s stating the conditions for null light scattering in certain directions by particles, such conditions have remained unquestioned. The increasing interest in scattering directionality by tuning the optical properties of materials demands a new analysis of this problem. In addition, as has been shown recently, one of Kerker's statements does not comply with the optical theorem. We propose corrected expressions for the null-scattering conditions that satisfy the optical theorem. (C) 2011 Optical Society of America OCIS codes: $290.1350,290.2558,290.2200$.
\end{abstract}

In recent years, the response of nanosystems to both the electric and the magnetic part of an incident electromagnetic field in the visible part of the spectrum has become an important topic. Systems with electric permittivity and magnetic permeability different from 1 have been analyzed from either a theoretical or an experimental point of view [1-3]. Although these optical properties cannot be observe $\bar{d}$ in natural materials, new engineered nanostructured materials, or metamaterials, have effective properties within such unusual intervals [4,, 5$]$. Scatterers with these optical properties may show nonconventional scattering behavior such as, for instance, negative refraction [6], which, for the case of bulk materials, could be useful for many applications: high-sensitivity biosensors, improved medical treatments, cloaked tools, or perfect lenses $[7,8]$. For the case of particles, we have focused our research on the possibility of controlling the angular distribution of the light scattered by a particle with unusual optical properties [9], following and generalizing the work presented by Kerker et al. [10].

Kerker and co-workers analyzed light scattering of a small spherical particle with arbitrary values for the relative electric permittivity $\left(\varepsilon_{r}\right)$ and magnetic permeability $\left(\mu_{r}\right)$. They showed that under certain conditions, the scattered intensity can be suppressed at given directions. In particular, they stated that if $\varepsilon_{r}=\mu_{r}$, light scattering in the backward direction is zero. As for forward scattering, it is inhibited when $\varepsilon_{r}=\left(4-\mu_{r}\right) /\left(2 \mu_{r}+1\right)$. These results have gained new interest because the control of the directionality of light scattering by tuning the optical constants is the key for several applications in optical communications [11]. However, these conditions, which we shall refer to as Kerker's conditions, have been controversial due to the violation of the optical theorem when the second condition is satisfied [12].

Alù and Engheta [12] proposed a correction in the expressions of the extinction cross section. By means of this correction, they showed that under the zero-forward condition, although the scattered intensity is not zero in the forward direction, it is minimum with respect to the other scattering angles and the optical theorem is fulfilled. In this Letter, we go one step further by including the radiative correction in the conditions stated by Kerker et al. [10], and we have obtained newly revised
Kerker's conditions. Thus, we can show that a small particle with optical constants $\left(\varepsilon_{r}, \mu_{r}\right)$ that satisfies these new conditions not only does not scatter either in the backward or the forward direction, but also with no violation of the optical theorem.

Following Kerker's steps [10] for a dipole-like particle in which the first-order Mie coefficients are dominant, the backscattering or the forward scattering equals zero when $a_{1}=b_{1}$ or $a_{1}=-b_{1}$, respectively. This implies that both the electric and magnetic polarizabilities are equal, that is $\alpha=\chi$, or they have an opposite sign, $\alpha=-\chi$. In [10], the authors used the Clausius-Mossotti relations to give form to the conditions. The Clausius-Mossotti relation for the electric polarizability is given by

$$
\alpha_{\mathrm{CM}}=3 V \frac{\varepsilon_{r}-1}{\varepsilon_{r}+2},
$$

while the magnetic polarizability $\left(\chi_{\mathrm{CM}}\right)$ has a similar expression in terms of $\mu_{r}$ instead of $\varepsilon_{r} . \varepsilon_{r}$ and $\mu_{r}$ are the relative optical constants of the scatterer, and $V$ is its volume.

If we now add the radiative correction [13] to this expression, we obtain

$$
\alpha_{\mathrm{CR}}=\frac{6 \pi V\left(\varepsilon_{r}-1\right)}{2 \pi\left(\varepsilon_{r}+2\right)-i V k^{3}\left(\varepsilon_{r}-1\right)},
$$

and a similar expression for $\chi_{\mathrm{CR}}$, but in terms of $\mu_{r}$ instead of $\varepsilon_{r}$.

By applying the previous equalities to these formulas, we can derive revised expressions for Kerker's conditions:

$$
\begin{gathered}
\text { zero-backward } \Rightarrow \varepsilon_{r}=\mu_{r}, \\
\text { zero-forward } \Rightarrow \varepsilon_{r}=\frac{\pi\left(4-\mu_{r}\right)-i V k^{3}\left(\mu_{r}-1\right)}{\pi\left(2 \mu_{r}+1\right)-i V k^{3}\left(\mu_{r}-1\right)},
\end{gathered}
$$

where $k$ is the wavenumber of the incident electromagnetic radiation.

The zero-backward condition is equal to that proposed by Kerker et al. [10], but the revised zero-forward 
condition presents an imaginary correction with respect to the Kerker's condition such that at least one of the optical constants should be complex. While the zerobackward condition can be satisfied for real values and no absorption, the zero-forward condition requires some degree of absorption in the scatterer. Because the imaginary part depends on $V$, it is directly related to the size and shape of the particle.

Figure 1 contains scattering diagrams corresponding to a spherical scatterer $(R=4.96 \mathrm{~nm})$ whose optical constants satisfy either Eqs. (3) or (4) illuminated by a plane wave with $\lambda=500 \mathrm{~nm}(R / \lambda \sim 0 . \overline{0} 1)$. The diagrams correspond to an incident electromagnetic field linearly polarized with the electric field parallel to the scattering plane (parallel polarization). We do not include the perpendicular polarization that produces similar angular distributions. For these calculations, we have used a computational tool based on Mie theory [14]. As can be seen, when the optical constants satisfy one of the previous relations, Eqs. (3) and (4), light scattering patterns show a clear zero at the backward or forward directions, respectively.

In a previous work [15], we showed that the second of Kerker's conditions, or the zero-forward condition, has an important exception when $\varepsilon_{r}=\mu_{r}=-2$, due to the excitation of two dipolar resonances, one electric and one magnetic. Although both conditions are satisfied simultaneously, only zero-backward scattering is actually observed. In their new form, the condition curves cross at the point

$$
\varepsilon_{r}=\mu_{r}=\frac{4 \pi+i V k^{3}}{-2 \pi+i V k^{3}} .
$$

This is a new form for the zero-forward exception.

The correction we introduce is a size correction in the sense that when $V \rightarrow 0$, the conditions tend to that of Kerker's original form. In Fig. 2, we show the evolution of $\mu_{r}$ (real and imaginary parts) as a function of $\varepsilon$, when they fulfill Eq. (4), for several particle sizes. As can be seen, while the real part of $\mu_{r}$ does not change as $R$ changes and it is similar to that obtained with the original Kerker's condition, the imaginary part becomes more negative as $R$ increases. Although the variation of the

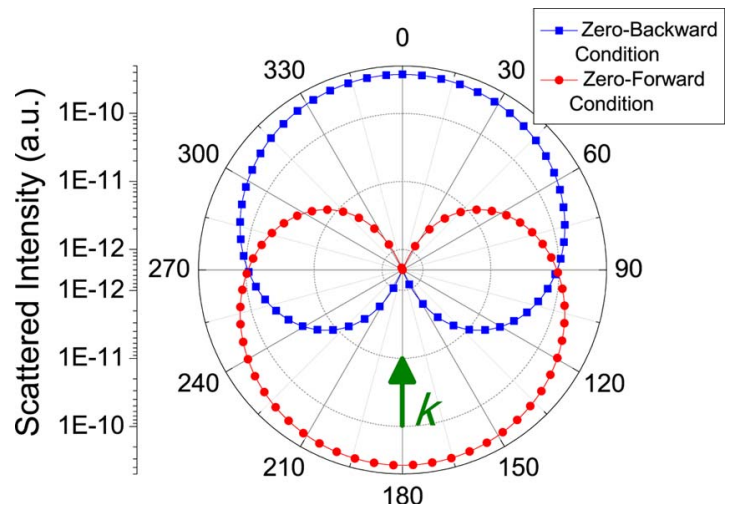

Fig. 1. (Color online) Scattering patterns of a spherical scatterer with $R=4.96 \mathrm{~nm}$, such that their optical constants satisfy either the new zero-forward (4) $\left[\left(\varepsilon_{r}, \mu_{r}\right)=(2,0.4-3.88 \times\right.$ $\left.\left.10^{-5} i\right)\right]$ or the zero-backward (3) $\left[\left(\varepsilon_{r}, \mu_{r}\right)=(2,2)\right]$ conditions. The arrow represents the direction of the incident field.

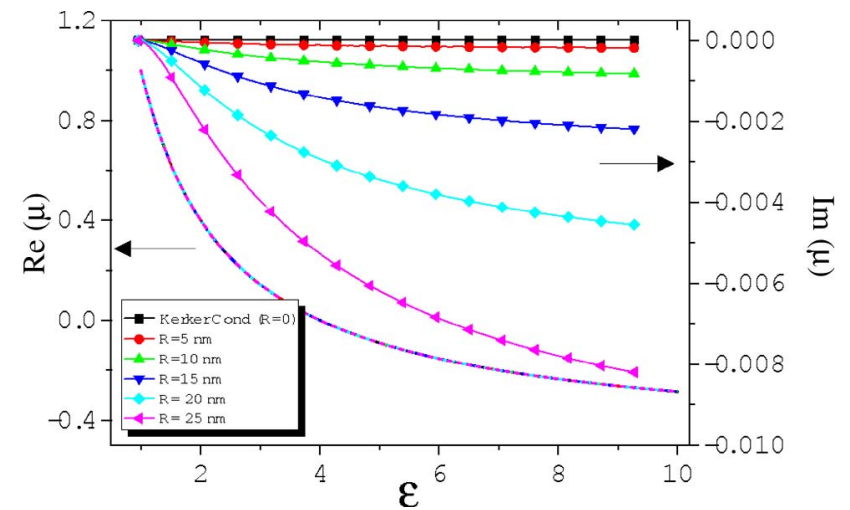

Fig. 2. (Color online) Real (continuos line, scale on the left) and imaginary (curves with symbols, scale on the right) part of the relative magnetic permeability as a function of the electric permittivity, following Eq. (ㄴ) ), for several particle sizes. For comparison, the values of $\bar{\mu}_{r}$ following the original Kerker's zero-forward condition are included.

imaginary part is quite small, it has important implications because it ensures that the optical theorem is satisfied, as will be shown.

The optical theorem establishes that the extinction cross section $\left(C_{\text {ext }}\right)$ is proportional to the scattering amplitude in the forward direction $\left[S\left(0^{\circ}\right)\right]$ in the following way [14]:

$$
C_{\mathrm{ext}}=\frac{4 \pi}{k^{2}} \operatorname{Re}\left\{S\left(0^{\circ}\right)\right\} .
$$

When the original zero-forward condition is satisfied, $S\left(0^{\circ}\right)=0$ and the extinction cross section is zero. The absorption cross section $\left(C_{\mathrm{abs}}\right)$ is also zero because the optical constants are real, but the scattering cross section $\left(C_{\mathrm{sca}}\right)$ is not zero because the scattered intensity has important values at other directions (see Fig. 1). These results are clearly inconsistent with $C_{\text {ext }}=C_{\text {sca }}+C_{\text {abs }}$.

However, in order to satisfy the zero-forward condition that we propose in Eq. (4), either $\varepsilon_{r}$ or $\mu_{r}$ must be complex if the other parameter is real and with negative values for the imaginary part, which gives rise to negative values of the absorption cross section or amplification cross section (see [16-18]). Scatterers with negative absorption are known as active objects, and they are the base of laser designs [19]. In Fig. 3 we plot the evolution of $C_{\mathrm{sca}}, C_{\mathrm{abs}}$, and $C_{\text {ext }}$ for a spherical particle $(R=$ $4.96 \mathrm{~nm})$ illuminated by a plane wave $(\lambda=500 \mathrm{~nm})$ as a function of the electric permittivity with a fixed value of the magnetic permeability $\left(\mu_{r}=0.4-3.88 \times 10^{-5} i\right)$. When the zero-forward condition proposed in Eq. (4) is fulfilled $\left[\left(\varepsilon_{r}, \mu_{r}\right)=\left(2,0.4-3.88 \times 10^{-5} i\right)\right]$, the extinction is zero, as stated by the optical theorem. At the same time, this is consistent with the relation $C_{\text {ext }}=C_{\text {sca }}+$ $C_{\text {abs }}$ because, at this point, $C_{\mathrm{sca}}=-C_{\mathrm{abs}}$.

In summary, we have analyzed the inconsistency created by the zero-forward-scattering condition proposed by Kerker et al. [10] and the optical theorem [14]. The introduction of the radiative correction in the generating expressions of the zero-forward condition produces a new form for such a condition, which complies with the optical theorem. In the limit $V \rightarrow 0$, the new expression tends to Kerker's. In addition, new conditions have size 


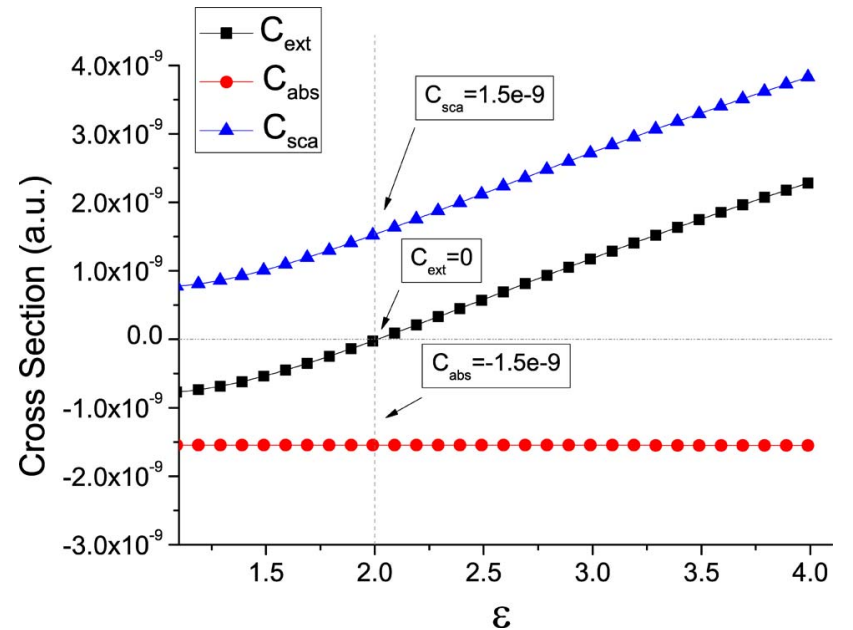

Fig. 3. (Color online) Evolution of the scattering, absorption (amplification), and extinction cross sections of a spherical particle $(R \simeq 5 \mathrm{~nm})$ as a function of $\varepsilon$ when the magnetic permeability is fixed $\left(\mu_{r}=0.4-3.88 \times 10^{-5} i\right)$. The pair $\left(\varepsilon_{r}, \mu_{r}\right)=\left(2,0.4-3.88 \times 10^{-5} i\right)$ satisfies the "new" zeroforward condition (4)).

limitations similar to those of Kerker's [9]. We have also analyzed the zero-backward condition, obtaining that the expression stated by Kerker et al. for zero-backward scattering is consistent and the radiative correction does not modify it.

The proposed conditions cannot be seen as a minor revision of the original ones, because the effect is influenced by the size of the scatterer. The significance of these results is also reinforced by considering systems of particles designed to achieve light directionality in the nanoscale. Finally, this Letter highlights again the importance of considering the radiative correction in studies of small particles.
This research has been supported by the Ministerio de Ciencia e Innovacion (MICINN) under project FIS200760158. R. Alcaraz de la Osa also thanks the MICINN for his FPU grant.

\section{References}

1. B. García-Cámara, F. Moreno, F. González, J. M. Saiz, and G. Videen, J. Opt. Soc. Am. A 25, 327 (2008).

2. Q. Wu and W. Park, Appl. Phys. Lett. 92, 153114 (2008).

3. A. N. Grigorenko, A. K. Geim, H. F. Gleeson, Y. Zhang, A. A. Firsov, I. Y. Khrushchev, and J. Petrovic, Nature 438, 335 (2005)

4. V. M. Shalaev, Nat. Photon. 1, 41 (2007).

5. H. Chen, C. T. Chan, and P. Sheng, Nat. Mater. 9, 387 (2010).

6. O. Hess, Nature 455, 299 (2008).

7. A. Alù and N. Engheta, Phys. Rev. Lett. 102, 233901 (2009).

8. A. V. Kabashin, P. Evans, S. Pastkovsky, W. Hendren, G. A. Wurtz, R. Atkinson, R. Pollard, V. A. Podolsky, and A. V. Zayats, Nat. Mater. 8, 867 (2009).

9. B. García-Cámara, J. M. Saiz, F. González, and F. Moreno, Opt. Commun. 283, 490 (2010).

10. M. Kerker, D.-S. Wang, and C. L. Giles, J. Opt. Soc. Am. 73, 765 (1983).

11. N. Engheta, Science 317, 1698 (2007).

12. A. Alù and N. Engheta, J. Nanophoton. 4, 041590 (2010).

13. B. T. Draine and P. J. Flatau, J. Opt. Soc. Am. A 11, 1491 (1994).

14. H. C. Van de Hulst, Light Scattering by Small Particles (Dover, 1981).

15. B. García-Cámara, F. González, F. Moreno, and J. M. Saiz, J. Opt. Soc. Am. A 25, 2875 (2010).

16. N. G. Alexopoulos and N. K. Uzunoglu, Appl. Opt. 17, 235 (1978).

17. M. Kerker, Appl. Opt. 18, 1180 (1979).

18. S. A. Ramakrishna and O. J. F. Martin, Opt. Lett. 30, 2626 (2005).

19. K. L. van der Molen, P. Zijlstra, A. Lagendijk, and A. P. Mosk, Opt. Lett. 31, 1432 (2006). 